\title{
Estudo da sorção de tensoativos orgânicos em argilas bentoníticas
}

\author{
(Study of organic surfactants sorption in bentonite clays)
}

\author{
D. L. da Silva, A. V. da Silva, H. S. Ferreira \\ Departamento de Engenharia de Materiais, Universidade Federal da Paraíba, PB, Brasil 58051-900 \\ darciely_lin@hotmail.com,amanda_vieirasilva@hotmail.com,hebersivini@gmail.com
}

\begin{abstract}
Resumo
Técnicas que envolvem a obtenção de argilas organofílicas vêm sendo estudadas por diversos pesquisadores, com o intuito de otimizar o processo de obtenção e garantir um produto de melhor qualidade. Todo estudo está embasado no fato dessas argilas apresentarem uma vasta gama de aplicações na engenharia. Porém, trabalhos que enfatizam o estudo químico desses processos atualmente são pouco frequentes. Desta forma, o principal objetivo deste trabalho foi estudar a sorção de tensoativos iônicos e não iônicos em argilas bentoníticas. No processo de organofilização utilizou-se a argila Brasgel, sendo organofilizada com dois tensoativos, o TA 50 (com $100 \%$ de matéria ativa) e o WB (com $81,26 \%$ de matéria ativa). Nas dispersões foram utilizadas cinco concentrações porcentuais em massa de diferentes argilas variando de $3,16 \%$ a $7,16 \%$. Cada concentração de argila foi organofilizada com quatro concentrações de tensoativo. As isotermas de sorção foram construídas através dos dados de termogravimetria e ajustadas ao modelo linear e de Freundlich. A partir das isotermas inferiu-se que os mecanismos envolvidos na sorção são a adsorção em multicamada (para os dois tensoativos utilizados) e a adsorção cooperativa (apenas para o WB), sendo o teor de argila com maior capacidade de sorção o de $3,16 \%$.
\end{abstract}

Palavras-chave: argila organofílica, tensoativo, sorção, isotermas.

\begin{abstract}
Techniques for obtaining organoclays have been studied by many researchers, intending to optimize the preparation process and to ensure a good quality product. These studies are based on the fact that such clays present a wide range of applications in engineering. However, works that emphasize the chemical study of these processes are less frequent. Thus, the main objective of this work was to study the sorption of ionic and non-ionic surfactants in bentonite clays. In organophilization process the Brasgel clay was used, with two surfactants, TA 50 (with 100\% active matter) and WB (with $81.26 \%$ active matter). In dispersions five different concentrations $(\% \mathrm{w} / \mathrm{w})$ of clay from $3.16 \%$ to $7.16 \%$ were used. Each clay concentration was organophilized with four different concentrations of surfactant. The sorption isotherms were constructed using the thermogravimetry data, and adjusted to the linear and Freundlich models. From the isotherms, it was inferred that the mechanisms involved in the sorption are multilayer adsorption (for two surfactants used) and cooperative adsorption (for WB); and the clay content of $3.16 \%$ had the higher sorption capacity.

Keywords: organoclay, surfactant, adsorption isotherms.
\end{abstract}

\section{INTRODUÇÃO}

Considera-se argila uma rocha constituída essencialmente por um grupo de minerais que recebe o nome de argilominerais; tanto as diferentes argilas como também cada uma das quatro dezenas de argilominerais têm nomenclaturas específicas. As argilas, além de serem constituídas por argilominerais, podem conter matéria orgânica, sais solúveis, partículas de quartzo, pirita, calcita, isto é, outros minerais considerados minerais acessórios ou contaminantes. Dependendo de sua mineralogia, as argilas apresentam propriedades que as caracterizam para uso em diversos processos de organofilização [1]. As argilas esmectíticas são materiais compostos por um ou mais argilominerais esmectíticos, sendo a montmorilonita o argilomineral mais abundante desse grupo; sua fórmula geral é $\mathrm{M}_{\mathrm{x}}\left(\mathrm{Al}_{4-\mathrm{x}} \mathrm{Mg}_{\mathrm{x}}\right) \mathrm{Si}_{8} \mathrm{O}_{20}(\mathrm{OH})_{4}$. Apresentam também em sua constituição alguns minerais acessórios, tais como quartzo, cristobalita, feldspatos e micas [2,3]. Sua estrutura é composta por unidades contendo duas folhas tetraédricas de sílica e por uma folha central octraédrica, onde um íon central $\mathrm{Al}^{3+}$ está rodeado por 6 oxigênios ou hidroxilas. A estrutura fundamental dos silicatos é formada pelo arranjo geométrico entre o silício e oxigênio. Pelo fato do silício possuir raio iônico de $0,40 \AA$, menor do que o do oxigênio que é $1,40 \AA$, o silício está rodeado por quatro oxigênios, originando assim uma configuração tetraédrica, cuja composição é $\mathrm{SiO}_{4}^{4}$, onde a carga (4-) resultante desse arranjo tetraédrico é compensada por ligações com íons positivos, como $\mathrm{Si}^{4+}, \mathrm{Fe}^{2+}$ e $\mathrm{Mg}^{2+}$, e isso faz com que seja restabelecido o equilíbrio eletrostático [4].

Pode-se chamar de bentonita uma rocha constituída essencialmente por um argilomineral montmorilonítico, que é formado pela desvitrificação e subsequente alteração química de um material vítreo, de origem ígnea, usualmente um tufo ou cinza vulcânica em ambientes alcalinos de circulação restrita de água [5]. As bentonitas podem ser sódicas ou cálcicas que, por sua vez, podem ser modificadas 
por intermédio de tensoativos e assim obtêm-se complexos argila-compostos orgânicos, denominados de argilas organofílicas. A síntese das argilas organofílicas ocorre de duas formas: por troca de íons (reação química de dupla troca), e íon-dipolo (moléculas orgânicas são adsorvidas na superfície da argila). É devido a essa propriedade que a argila bentonítica tem um grande poder adsorvente, especialmente de adsorver íons em solução, e são argilas bastante utilizadas no processo de organofilização e isso se deve às suas características $[1,6]$. O processo de organofilização foi comumente estudado com valores fixos de tensoativos da ordem $110 \mathrm{meq} / 100 \mathrm{~g}$ de argila seca [7], em dispersões de 4\% em massa. Outros estudos fixaram proporção entre a massa de tensoativo iônico e a massa de argila em intervalos entre $63 \%$ e $123 \%$ em dispersões de $4,86 \%$ em massa $[8,9]$; mais recentemente passou-se a usar teores de tensoativo entre $25 \%$ e $45 \%$ em relação à massa de argila [10]. A capacidade de troca de íons na argila foi considerada, neste trabalho, fator determinante para quantificação dos tensoativos durante os processos de organifilização e no caso dos iônicos. Os percentuais de massa uma vez convertidos para meq são comparáveis com a CTC (capacidade de troca catiônica) da argila o que permite a obtenção da estequiometria [11]. A opção de iniciar os estudos com um teor levemente superior ao valor da CTC justifica-se ao fato de manter a solução sempre saturada o que, em tese, garante que não haja posições de troca disponíveis ao final do processo [11]. No caso dos não iônicos, repete-se a mesma proporção usada nos iônicos.

O mecanismo de sorção é relativamente bem conhecido, porém o estudo da sorção/troca catiônica de tensoativo, tanto iônicos quanto não iônicos, na argila bentonítica e sua quantificação não é algo amplamente estudado. A sorção é um processo que envolve a adsorção, quimissorção (adsorção química), absorção e troca iônica, sendo a adsorção o processo no qual o soluto adere às superfícies do adsorvente, especialmente argilominerais e matéria orgânica, por possuírem cargas desequilibradas nas superfícies das partículas ou substituições iônicas (substituição isomórfica) na estrutura cristalina dos minerais ou de quebra de ligações nas estruturas moleculares especialmente nas extremidades $[12,13]$. A absorção é o fenômeno que ocorre quando o soluto pode difundir dentro do adsorvente (sólido) na matriz porosa. São fenômenos parecidos, porém o que distingue um do outro é que a absorção se trata de um fenômeno químico que permeia o volume de outra, como uma esponja que absorve água; já a adsorção é um fenômeno de superfície, que ocorre quando as moléculas reagem quimicamente com a superfície, podendo em alguns casos ocorrer rompimento e formação de ligações. Quando se trata de transporte de massa de uma fase para a outra, esses fenômenos são generalizados e chamados de sorção [14].

A sorção pode ser determinada experimentalmente medindo o quanto de soluto pode ser sorvido pelo substrato. Essa por sua vez é geralmente quantificada através do coeficiente de distribuição $K_{d}$, que pode ser determinado por meio da análise das isotermas que expressam a relação entre quantidade de soluto sorvido e a concentração de soluto em massa na fase líquida (concentração no equilíbrio) [14-16]. Com intuito de melhorar o ajuste das curvas (isotermas), várias equações foram desenvolvidas, sendo as mais conhecidas e frequentemente utilizadas a de Freundlich, de Langmuir e a isoterma linear, que por sua vez é um caso especial da isoterma de Freundlich. Vale salientar que diversos fatores influenciam na sorção, entre eles estão a CTC da argila, a mineralogia da argila, pois a espécie de argilomineral presente na argila é um fator de forte influência na CTC, concentração de soluto e tipo de soluto $[10,17]$. Este trabalho tem como objetivo estudar a sorção/troca catiônica de tensoativos iônicos e não iônicos em argilas bentoníticas, variando-se as concentrações de argilas e tensoativos, e analisar de forma quantitativa quanto de tensoativo orgânico foi sorvido durante o processo de organofilização.

\section{EXPERIMENTAL}

Materiais: utilizaram-se a argila Brasgel PA (BG) do fabricante Bentonit União Nordeste, proveniente do município de Boa Vista, PB, o tensoativo Praepagem $\mathrm{WB}^{\circledR}$ (cloreto de diestearil dimetil amônio), com $81,26 \%$ de matéria ativa, fornecido pelo fabricante Clariant (S. Paulo, SP), a amina etoxilada de grau 5 TA $50^{\circledR}$ com $100 \%$ de matéria ativa, da empresa Oxiteno (Mauá, SP). As amostras de argilas foram caracterizadas física e mineralogicamente através dos seguintes métodos: capacidade de troca catiônica (CTC) e difração de raios X (DRX).

Capacidade de troca de cátions (CTC): a CTC das argilas foi determinada através da técnica de adsorção de azul de metileno [18], calculada utilizando a Equação A:

$$
\text { C.T.C }=\frac{\text { V.C.100 }}{\text { Massa de amostra seca }(g)}
$$

onde, $\mathrm{V}$ é o volume total e $\mathrm{C}$ é a concentração de azul de metileno (em normalidade).

Difração de raios $X$ : os difratogramas de raios $\mathrm{X}$ das argilas foram obtidos por meio de um difratômetro Siemens D-5000. Inicialmente as argilas organofílicas e as argilas bentoníticas foram passadas na peneira $\mathrm{ABNT} \mathrm{n}^{\circ} 200(74 \mu \mathrm{m})$ e, em seguida, foram prensadas manualmente em um portaamostra de Al. Utilizaram-se faixas de $2 \theta$ de $1,5^{\circ}$ a $10^{\circ}$ para as argilas organofílicas e de $5^{\circ}$ a $60^{\circ}$ para as argilas bentoníticas, com radiação $\mathrm{K} \alpha$ do $\mathrm{Cu}$, correspondente a 1,5418 $\AA$ de comprimento de onda, e corrente de 30 e $40 \mathrm{~mA}$.

Processo de organofilização: a argila Brasgel foi organofilizada com dois tensoativos, o TA 50 (com 100\% de matéria ativa) e o WB (com $81,26 \%$ de matéria ativa). Para preparações das dispersões foram utilizadas cinco concentrações (\% em massa) diferentes de argila, onde essas foram variadas de 3,16\% a 7,16\%. Cada concentração de argila foi organofilizada como quatro concentrações diferentes de tensoativo, conforme as Tabelas I e II. 
Tabela I - Relação entre massa de WB adicionada na argila e massa de argila seca. [Table I - Relationship between WB mass added in clay and mass of dry clay.]

\begin{tabular}{cccc}
\hline Teor de WB & $\begin{array}{c}\text { Quantidade de WB } \\
(\mathrm{g}) / 100 \mathrm{~g} \text { de argila }\end{array}$ & $\begin{array}{c}\text { meq de WB/100 g de } \\
\text { argila seca }\end{array}$ & $\begin{array}{c}\text { Razão entre a CTC da } \\
\text { argila e meq de WB }\end{array}$ \\
\hline Teor 1 & 40,0 & 105,0 & 1,25 \\
Teor 2 & 47,0 & 122,0 & 1,46 \\
Teor 3 & 53,0 & 139,0 & 1,67 \\
Teor 4 & 60,0 & 157,0 & 1,88 \\
\hline
\end{tabular}

Tabela II - Relação entre massa de TA 50 adicionada na argila e massa de argila seca.

[Table II - Relationship between mass of TA 50 added in clay and mass of dry clay.]

\begin{tabular}{cc}
\hline Teor de TA50 & Quantidade de TA50 $(\mathrm{g}) / 100 \mathrm{~g}$ de argila \\
\hline Teor 1 & 40,0 \\
Teor 2 & 47,0 \\
Teor 3 & 53,0 \\
Teor 4 & 60,0 \\
\hline
\end{tabular}

Organofilização da argila: para a preparação das dispersões foram utilizados teores de 3,16\% a 7,16\% em massa da argila bentonítica, que foram dispersos em água destilada a $80^{\circ} \mathrm{C}$ (temperatura da água antes de adicionar a argila); a mistura foi mantida em agitação a uma velocidade de aproximadamente $1900 \mathrm{rpm}$, durante 20 min (necessário para que ocorra a dispersão da argila). Após o processo de dispersão o tensoativo foi adicionado aos poucos, variando seu teor conforme a Tabela I para o tensoativo WB e conforme a Tabela II para o TA 50, que por se tratar de um tensoativo não iônico não foi levada em conta a CTC. $\mathrm{O}$ pH do meio foi controlado com $\mathrm{HCl} 2 \mathrm{~N}$ até $\mathrm{pH}$ 7,0 (neutro). A agitação foi mantida por 20 min e, em seguida, filtrou-se a dispersão em funil de Büchner, onde este estava acoplado à bomba a vácuo. Decorrida a etapa de filtração, a argila organofílica foi seca a $66{ }^{\circ} \mathrm{C}$ em estufa com ventilação por um período de $48 \mathrm{~h}$; em seguida as argilas foram desagregadas manualmente em um almofariz e passadas em peneira ABNT n ${ }^{\circ} 200(74 \mu \mathrm{m})$.

Isotermas de sorção: o procedimento usado para o estudo da sorção foi o ensaio em lote, que consistiu na colocação de uma determinada massa de argila em contato e agitação contínua com soluções contendo quantidades crescentes de tensoativo, onde a concentração foi variada como mostrado na Tabela I para o tensoativo iônico (WB) e na Tabela II para o tensoativo não iônico (TA 50). Para ambos, levou-se em consideração a relação a $100 \mathrm{~g}$ de argila seca utilizada para organofilização da argila. A dispersão foi filtrada e a concentração de tensoativo adsorvido na superfície da argila foi determinada por meio da análise termogravimétrica (TG). A quantidade de tensoativo adsorvido na argila foi determinada usando a Equação B [19]:

$$
\mathrm{S}=\frac{\left(\mathrm{C}_{0}-\mathrm{C}_{\mathrm{e}}\right) \cdot \mathrm{V} \cdot 1000}{\mathrm{M}}
$$

onde, $\mathrm{S}$ é a massa de soluto adsorvido por unidade de massa do sólido $(\mu \mathrm{g} / \mathrm{g}), \mathrm{C}_{0}$ a concentração inicial da solução $(\mathrm{mg} / \mathrm{L})$, $\mathrm{C}_{\mathrm{e}}$ a concentração final da solução em equilíbrio $(\mathrm{mg} / \mathrm{L}), \mathrm{V}$ o volume da solução que foi utilizada no experimento (L) e M é a massa de sólido ( $\mathrm{g}$ ). Uma isoterma de sorção foi obtida, colocando-se em um diagrama cartesiano os valores de $\mathrm{S}$ e $\mathrm{C}_{\mathrm{e}}$, que foi calculada subtraindo a quantidade de tensoativo sorvido na amostra de argila pela quantidade que pesava (considerando o que restou de tensoativo no béquer durante a adição), baseando-se na quantidade de tensoativo adicionado no processo de organofilização. A modelagem da sorção foi obtida através do ajuste de parâmetros de modelos estatísticos conhecidos aos dados experimentais, usando as seguintes equações:

-Linear: este modelo prevê em concentrações infinitamente crescentes a sorção do soluto e pode ser representado pela Equação C [19]:

$$
\mathrm{S}=\mathrm{K}_{\mathrm{d}} \cdot \mathrm{C}_{\mathrm{e}}
$$

onde, $\mathrm{K}_{\mathrm{d}}$ corresponde ao coeficiente de distribuição expresso pelo coeficiente angular da reta $\left(\mathrm{cm}^{3} / \mathrm{g}\right)$.

-Freundlich: esta modelagem surgiu como uma alternativa para melhor representar os processos sorcivos que possuem uma forma curvilínea, quando se plota o gráfico $\mathrm{S}_{\mathrm{X} \mathrm{C}} \mathrm{e}_{\mathrm{e}}$, e pode ser representada pela Equação D [19]:

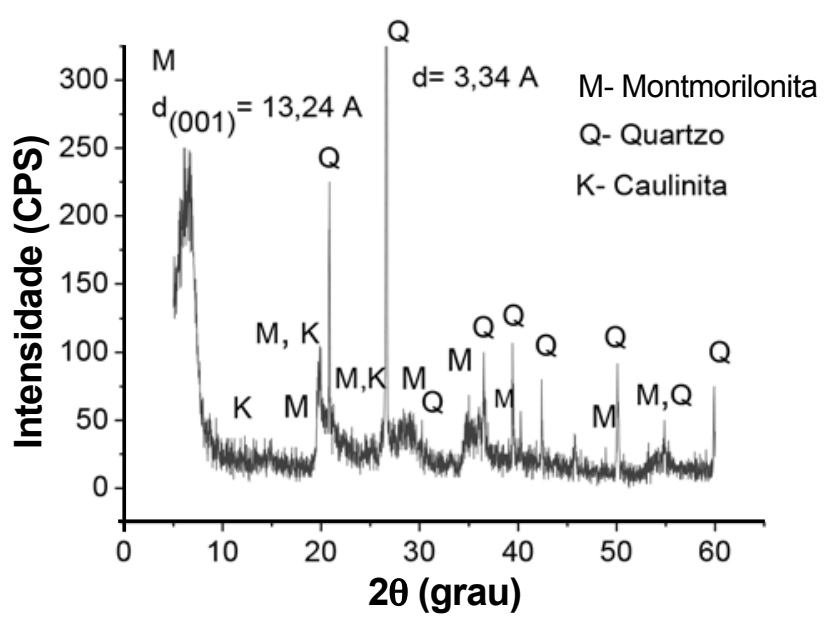

Figura 1: Difratograma de raios $\mathrm{X}$ da argila Brasgel não organofilizada.

[Figure 1: X-ray diffraction pattern of not organophilizated Brasgel clay.] 


$$
\mathrm{S}=\mathrm{K}_{\mathrm{f}} \mathrm{C}_{\mathrm{e}}{ }^{\mathrm{N}}
$$

onde, $\mathrm{K}_{\mathrm{f}}$ corresponde ao coeficiente de partição de Freundlich $\left(\mathrm{cm}^{3} / \mathrm{g}\right)$ e $\mathrm{N}$ é considerado como sendo o coeficiente exponencial de Freundlich; através do valor de $\mathrm{N}$ é possível especificar o tipo de sorção, pois quando $\mathrm{N}>1$ considera-se a sorção favorável, para $\mathrm{N}<1$ a sorção é dita desfavorável e quando $\mathrm{N}=1$ a sorção se torna igual ao modelo linear [16, 19].

\section{RESULTADOS E DISCUSSÃO}

Capacidade de troca de cátions (CTC): a argila Brasgel apresentou um resultado de CTC de 84,0 meq/100 g, sendo
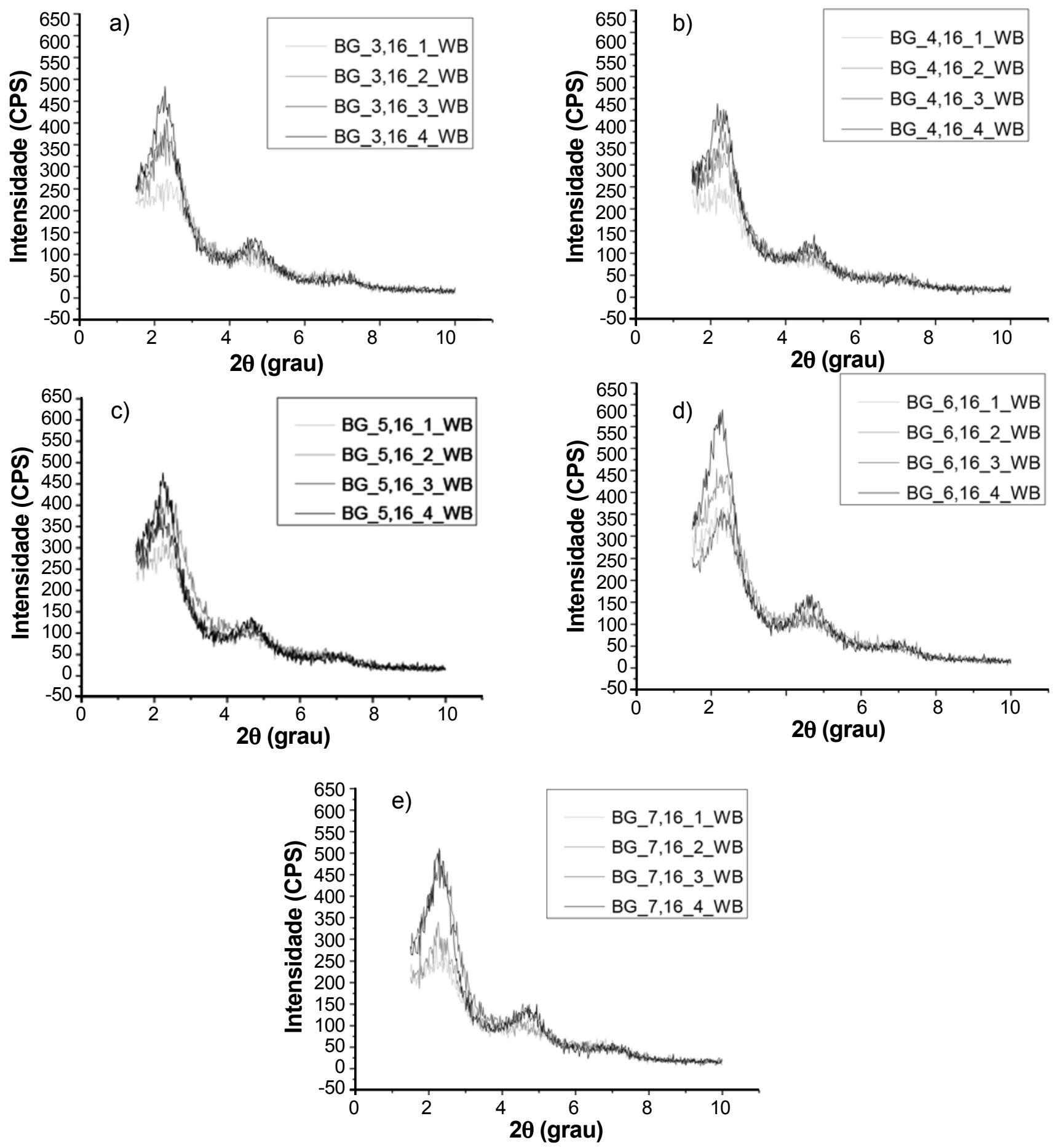

Figura 2: Difratogramas de raios X da argila Brasgel organofilizada com tensoativo WB em concentração (\% em massa) de: (a) 3,16\%; (b) $4,16 \%$; (c) $5,16 \%$; (d) $6,16 \%$; (e) $7,16 \%$.

[Figure 2: X-ray diffraction patterns of the Brasgel clay organophilizated with surfactant WB at concentration (w/w) of: (a) 3.16\%; (b) $4.16 \%$; (c) $5.16 \%$; (d) $6.16 \%$; (e) $7.16 \%$. 

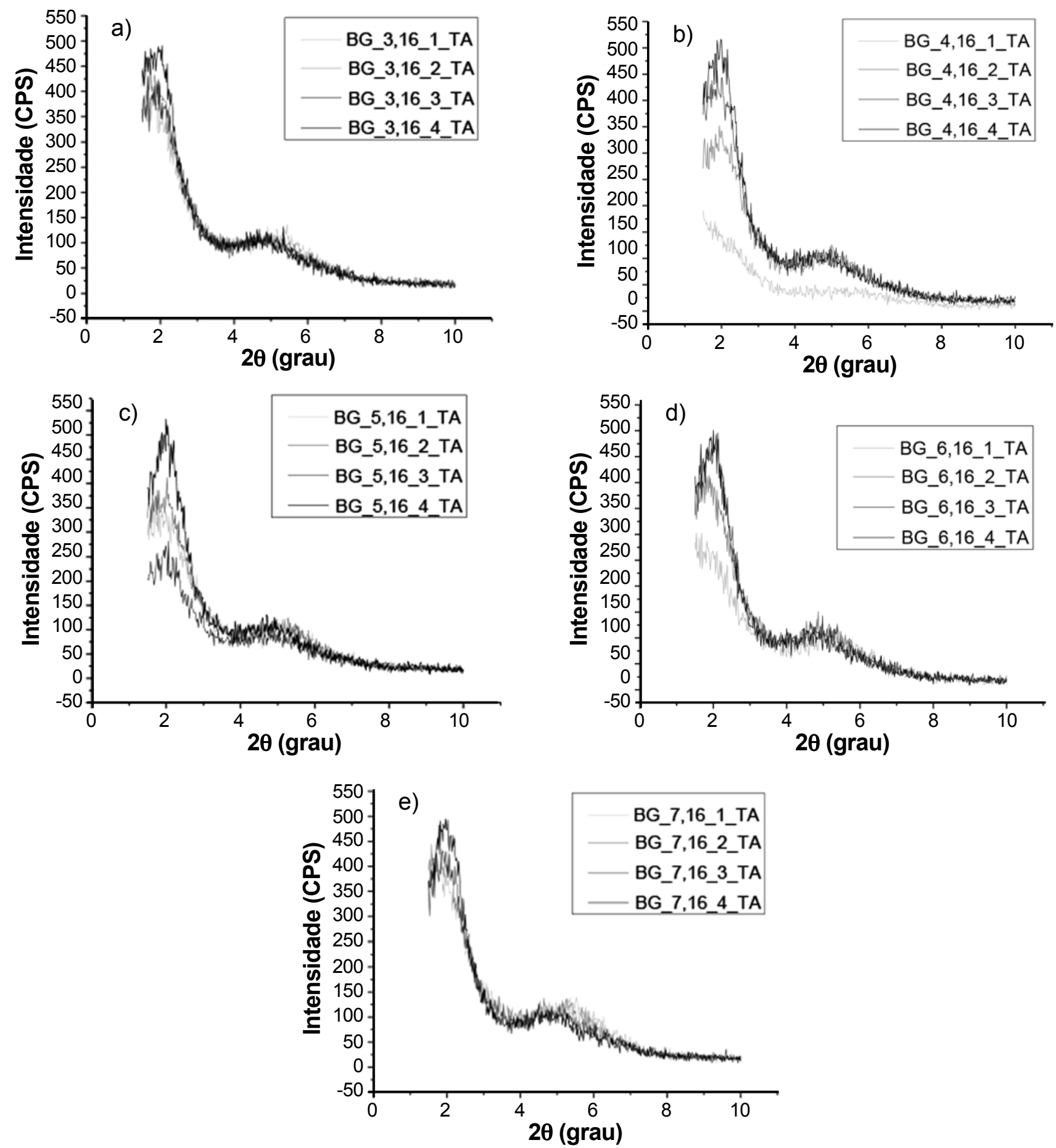

Figura 3: Difratogramas de raios X da argila Brasgel organofilizada com tensoativo TA 50 em concentração (\% em massa) de: (a) 3,16\%; (b) $4,16 \%$; (c) $5,16 \%$; (d) $6,16 \%$; (e) $7,16 \%$.

[Figure 3: X-ray diffraction patterns of the Brasgel clay organophilizated with surfactant TA 50 at concentration (w/w) of: (a) 3.16\%; (b) $4.16 \%$; (c) $5.16 \%$; (d) $6.16 \%$; (e) $7.16 \%$.]

esse um resultado similar aos encontrados na literatura [20].

Difração de raios $X(D R X)$ : a Fig. 1 apresenta o difratograma da argila que foi objeto de estudo. No difratograma pode-se observar a presença da esmectita através de diversos picos, sendo evidente um pico caracterizado pela distância interplanar de 13,24 $\AA$ que é referente ao plano (001), existindo ainda outros picos com menor intensidade apresentando distâncias de 13,09 e $12,93 \AA$ A A presença do quartzo na argila é caracterizada com ênfase pela distância interplanar de $3,34 \AA$, porém vale salientar que há a presenças de outros picos característicos do quartzo caracterizados pelas distâncias de 4,26 e 2,96 ̊. Além do quartzo que é considerado como impureza, podese identificar também a caulinita, onde o pico com maior destaque é identificado pela distância de 7,15 ̊̊; outros picos característicos da caulinita podem ser identificados em 4,47 

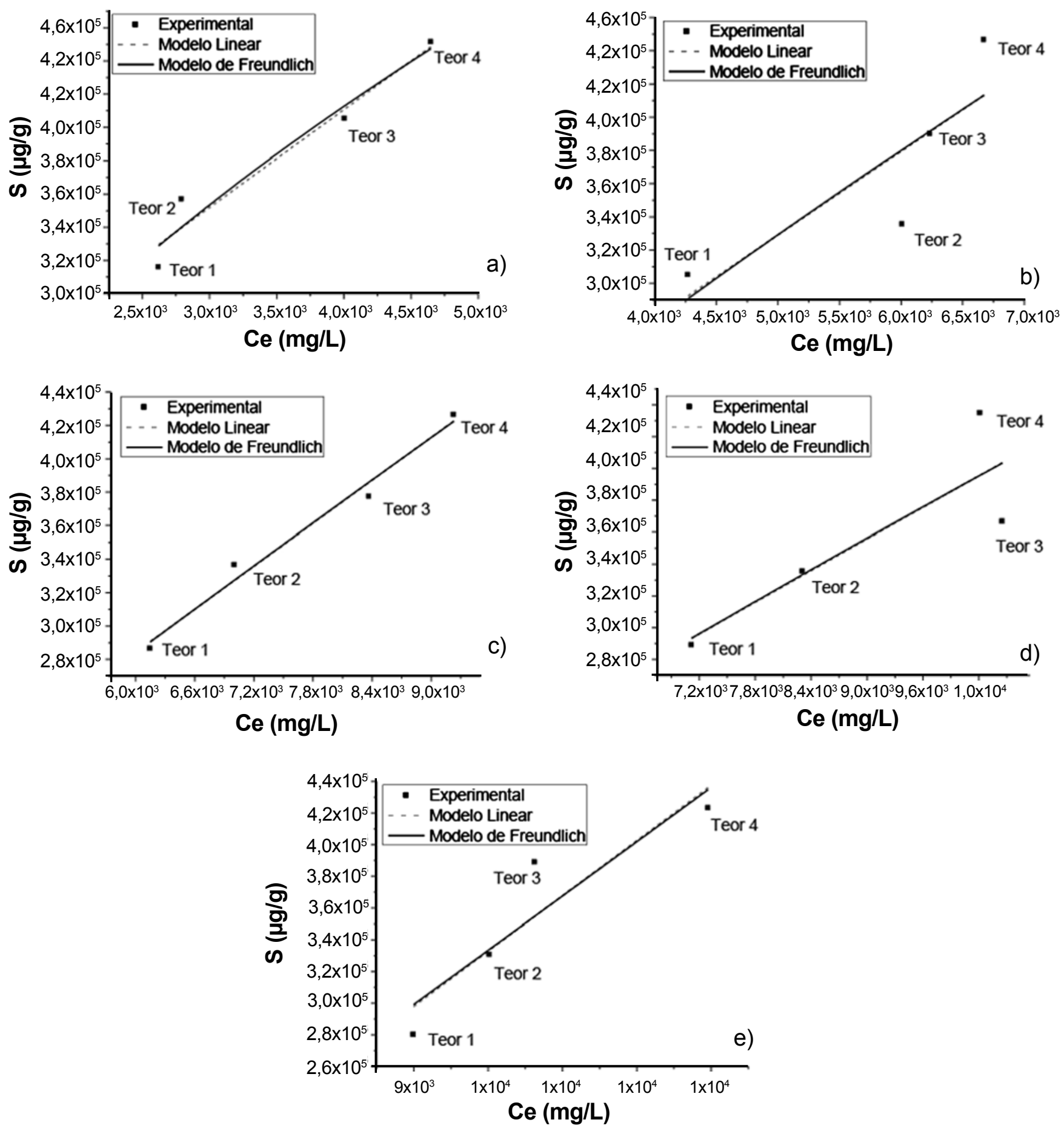

Figura 4: Isotermas de sorção do tensoativo WB incorporado na argila Brasgel de concentração (\% em massa) de: (a) 3,16\%; (b) 4,16\%; (c) $5,16 \%$; (d) $6,16 \%$; (e) $7,16 \%$.

[Figure 4: WB surfactant sorption isotherms incorporated in Brasgel clay at concentration (w/w) of: (a) 3.16\%; (b) 4.16\%; (c) 5.16\%; (d) $6.16 \%$; (e) $7.16 \%$.

e 5,91 $\AA$, sendo ambos impurezas normalmente encontradas em argilas bentoníticas $[8,15]$.

\section{Atributos das argilas organofilicas}

Os difratogramas da Fig. 2, referentes a argila organofílica obtida através da organofilização da argila Brasgel com o tensoativo WB, indicam as variações no ângulo e na distância $\mathrm{d}_{001}$. Observa-se nos difratogramas da Fig. 2 a presença de um conjunto de picos diferenciados. Esses picos evidenciam que, provavelmente, há duas expansões relacionadas com duas orientações de moléculas e que provavelmente parte dos espaçamentos interplanares não foram intercalados pelo cátion do tensoativo. Indicam também que algumas camadas são mais facilmente intercaladas com o tensoativo e que outras são mais difíceis de serem intercaladas [8]. De 
acordo com os difratogramas da Fig. 2, pode-se inferir que após a organofilização a presença do tensoativo provocou um deslocamento do pico referente ao plano cristalográfico (001) para ângulos mais baixos, que consequentemente caracteriza aumento do espaçamento basal [8]. Apenas as argilas de concentração 3,16\%, 4,16\% e 7,16\% apresentaram aumento de intensidade que variou de acordo com o aumento da quantidade de tensoativo adicionado; isto é, quando a concentração de argila não foi alterada e variou-se a quantidade de tensoativo, apenas nas argilas citadas houve um aumento na intensidade do pico de reflexão da esmectita com o aumento do teor da concentração de tensoativo, enfatizando que a estrutura da argila tornou-se mais organizada com o aumento de tensoativo. Vale salientar que para todas as concentrações de argila estudadas, a intensidade do pico de reflexão da esmectita foi maior quando se utilizou o tensoativo no Teor 4.

Na Fig. 3 são apresentados os difratogramas da argila Brasgel nas concentrações estudadas, organofilizada com tensoativo TA 50. Um fato semelhante é observado na Fig. 3 quando comparado com a Fig. 2, a presença de mais de um pico nos difratogramas, porém diferentemente da argila quando organofilizada com $\mathrm{WB}$, as que foram organofilizadas com o TA 50 apresentaram apenas um conjunto de picos. Desse fato também pode-se inferir que o TA 50 é um tensoativo não iônico e é intercalado na argila por meio de adsorção. O tipo de tensoativo é uma variável influente na organofilização [9]. Com relação à intensidade dos picos, que fornece informações sobre a organização da estrutura cristalina da argila, apenas as argilas que foram organofilizadas na concentração $4,16 \%$, $6,16 \%$ e $7,16 \%$ apresentaram um aumento gradativo com o aumento da concentração de tensoativo, ou seja, o aumento da concentração de tensoativo favoreceu a organização da estrutura cristalina da argila; a intensidade das demais procedeu de forma aleatória. A argila Brasgel nas concentrações de $4,16 \%$ e $6,16 \%$, quando organofilizada com o Teor 1 de TA 50, não apresentou picos definidos para inferir se a adição de tensoativo favoreceu a organização da estrutura cristalina. Mas, vale destacar que quando se utilizou o tensoativo na concentração de $60 \mathrm{~g} / 100 \mathrm{~g}$ de argila seca, para ambas as concentrações de argila, a intensidade do pico foi maior.

\section{Isotermas de sorção}

Na Fig. 4 são representadas as isotermas de sorção da argila Brasgel organofilizada com o tensoativo WB. Ao analisar estas isotermas, infere-se que elas são classificadas como isotermas do tipo $\mathrm{C}$ e $\mathrm{L}$, que são muito usadas para materiais geológicos [19]. Para todas as concentrações de argila houve um aumento da sorção de tensoativo com a elevação da quantidade inicial do mesmo; de acordo com a CTC, a capacidade máxima de troca de cátions dessa argila é equivalente a uma sorção de $3,21 \times 10^{5} \mu \mathrm{g} / \mathrm{g}$, porém se observam nas isotermas que foi possível sorver quantidades superiores a essa. Isto contradiz que é por meio da capacidade de troca catiônica (CTC) dos argilominerais que se determina a capacidade de sorção de compostos, sendo essa diretamente relacionada com o $\mathrm{pH}$, apresentando sua máxima sorbância em $\mathrm{pH}$ entre 1 e 5 . É possível quantificar a CTC baseando-se no número de mol possível de ser fixado em $100 \mathrm{~g}$ de argila seca [21,22], fato que pode ser explicado levando-se em consideração que dados experimentais que são ajustados ao modelo de Freundlich admitem adsorção em multicamadas. Esse fato pode ainda estar relacionado com a interação lateral entre o monômero adsorvido, o que resulta em agregação do tensoativo na superfície da argila que consequentemente provoca um aumento da área de adsorção [23-25]. Provavelmente à medida que se aumentou o teor de argila, esse aumento dificultou a difusão do tensoativo para

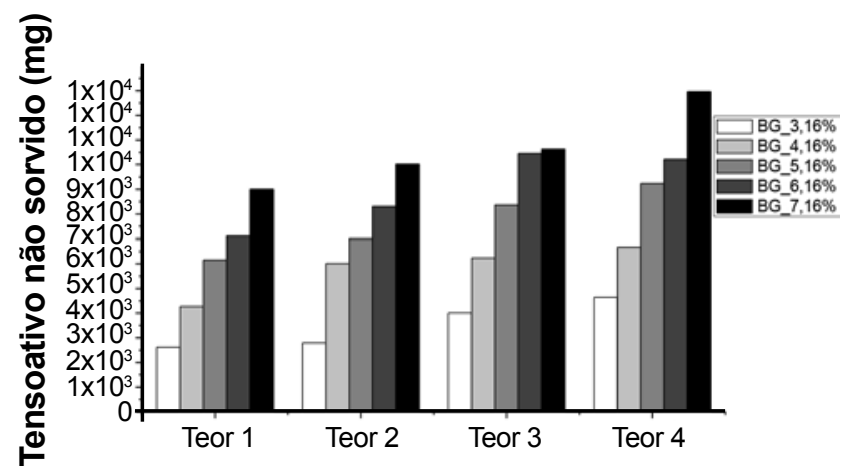

Figura 5: Quantidade de WB que não foi incorporado na argila Brasgel.

[Figure 5: Amount of WB that was not incorporated into Brasgel clay.]

Tabela III - Parâmetros de sorção para a argila Brasgel organofilizada com tensoativo WB obtidos pela equação linear e de Freundlich.

[Table III - Sorption parameters for Brasgel clay organophilizated with surfactant WB obtained by linear and Freundlich equation.]

\begin{tabular}{ccccccc}
\hline & & BG_3,16\% & BG_4,16\% & BG_5,16\% & BG_6,16\% & BG_7,16\% \\
\hline \multirow{2}{*}{ Linear } & $\mathrm{R}^{2}$ & 0,924 & 0,584 & 0,973 & 0,610 & 0,792 \\
& $\mathrm{~K}_{\mathrm{d}}\left(\mathrm{cm}^{3} / \mathrm{g}\right)$ & 58,7 & 50,3 & 42,9 & 33,1 & 34,9 \\
\multirow{5}{*}{ Freundlich } & $\mathrm{R}^{2}$ & 0,924 & 0,571 & 0,974 & 0,613 & 0,791 \\
& $\mathrm{~K}_{\mathrm{f}}\left(\mathrm{cm}^{3} / \mathrm{g}\right)$ & 4764,8 & 401,2 & 92,9 & 189,0 & 26,6 \\
& $\mathrm{~N}$ & 0,538 & 0,788 & 0,922 & 0,828 & 1,024 \\
\hline
\end{tabular}



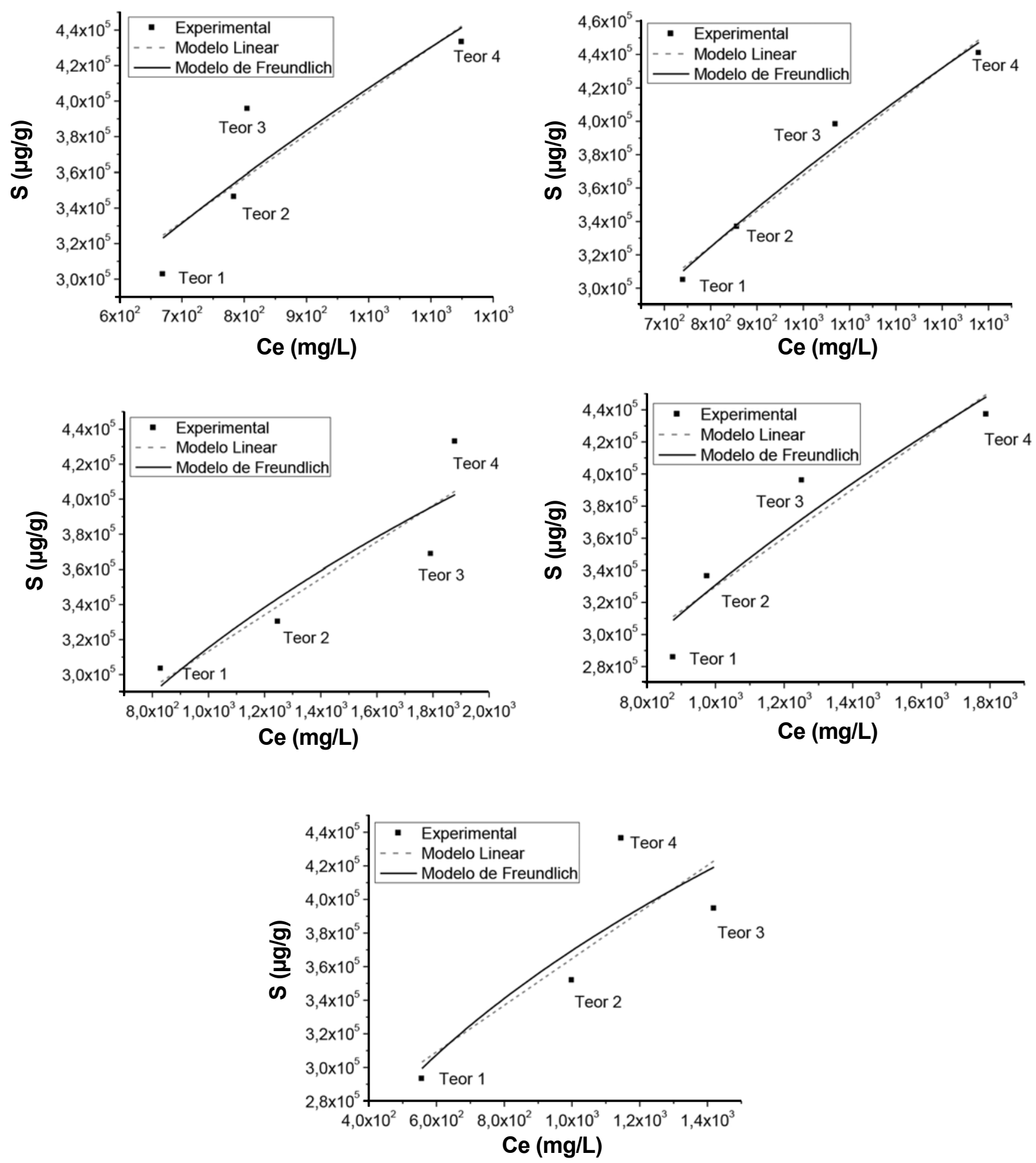

Figura 6: Isotermas de sorção do tensoativo TA50 incorporado na argila Brasgel de concentração (\% em massa) de: (a) 3,16\%; (b) $4,16 \%$; (c) $5,16 \%$; (d) $6,16 \%$; (e) $7,16 \%$.

[Figure 6: TA 50 surfactant sorption isotherms incorporated in Brasgel clay at concentration (w/w) of: (a) 3.16\%; (b) 4.16\%; (c) $5.16 \%$; (d) $6.16 \%$; (e) $7.16 \%$.]

os sítios de adsorção, pois fica mais difícil de ocorrer a troca de cátions entre o tensoativo e a argila, devido ao aumento do teor de argila que possivelmente provoca uma diminuição da região disponível para troca; se essa região diminui com o aumento do teor de argila, menos tensoativo será sorvido.
Os parâmetros desses modelos podem ser encontrados na Tabela III.

Dois coeficientes são importantes para escolha do modelo que melhor se ajustou aos dados experimentais, $\mathrm{o} \mathrm{R}^{2}$ (coeficiente de determinação) e o $\mathrm{N}$, sendo o $\mathrm{N}$ um coeficiente 
Tabela IV - Parâmetros de sorção para a argila Brasgel organofilizada com tensoativo TA 50 obtidos pela equação linear e de Freundlich.

[Table IV - Sorption parameters for Brasgel clay organophilizated with surfactant TA 50 obtained by linear and Freundlich equation.]

\begin{tabular}{lllllll}
\hline & & BG_3,16\% & BG_4,16\% & BG_5,16\% & BG_6,16\% & BG_7,16\% \\
\hline \multirow{2}{*}{ Linear } & $\mathrm{R}^{2}$ & 0,689 & 0,952 & 0,736 & 0,810 & 0,504 \\
& $\mathrm{~K}_{\mathrm{d}}\left(\mathrm{cm}^{3} / \mathrm{g}\right)$ & 244,9 & 213,8 & 103,8 & 151,7 & 138,8 \\
& $\mathrm{R}^{2}$ & 0,716 & 0,968 & 0,698 & 0,850 & 0,560 \\
Freundlich & $\mathrm{K}_{\mathrm{f}}\left(\mathrm{cm}^{3} / \mathrm{g}\right)$ & 7565,4 & 6414,1 & 2172,2 & 9042,6 & 3078,4 \\
& $\mathrm{~N}$ & 0,577 & 0,587 & 0,387 & 0,521 & 0,360 \\
\hline
\end{tabular}

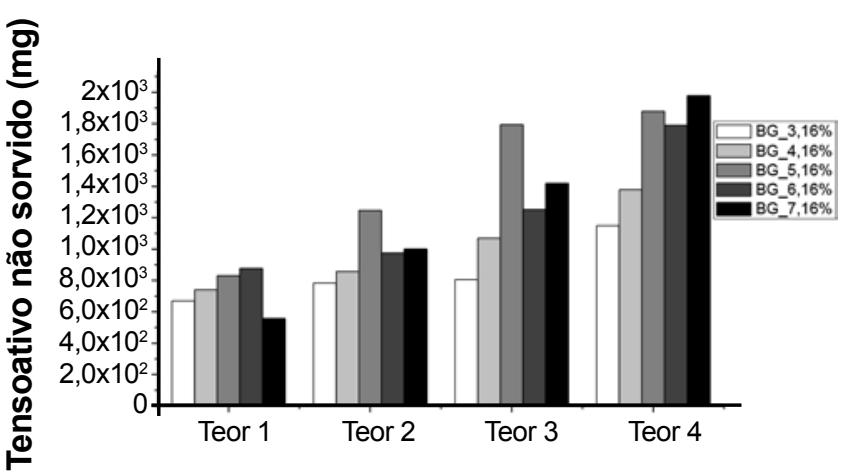

Figura 7: Quantidade de TA 50 que não foi incorporado na argila Brasgel.

[Figure 7: Amount of TA 50 that was not incorporated into Brasgel clay.]

importante, pois, observando a equação de Freundlich (Equação $\mathrm{C}$ ), se o $\mathrm{N}$ for igual a 1 ou muito próximo de 1 a Equação $\mathrm{C}$ se torna linear. Desta forma, convém afirmar que de acordo com o $\mathrm{R}^{2}$, os dados experimentais das argilas BG_4,16\% e BG_7,16\% se ajustaram melhor ao modelo linear, no entanto os dados experimentais das argilas BG_3,16\%, BG_5,16\% e BG_6,16\% se ajustaram melhor ao modelo de Freundlich. Analisando os valores de $\mathrm{N}$ dispostos na Tabela III, é possível inferir que o valor de $\mathrm{N}$ foi aproximadamente 1 apenas para a argila $\mathrm{BG}_{-} 7,16 \%$; para as demais, o valor de $\mathrm{N}$ foi abaixo de 1, indicando que a sorção é desfavorável. $\mathrm{O} \mathrm{K}_{\mathrm{d}}$ e $\mathrm{K}_{\mathrm{f}}$ são constantes que quantificam a distribuição do soluto entre as fases aquosa e sólida, desta forma maiores valores dessas constantes representam uma maior transferência de tensoativo da solução para a argila [17]. De acordo com a Tabela III, observa-se que a argila BG_3,16\% apresentou um valor maior de $\mathrm{K}_{\mathrm{d}}$ e $\mathrm{K}_{\mathrm{f}}$; já para as argilas BG_4,16\%, BG_5,16\%, BG_6,16\% e BG_7,16\% os valores de $K_{d}$ e $K_{f}$ foram menores e consequentemente a sorção de tensoativo foi menor. Pode-se observar melhor esse fato através das isotermas apresentadas na Fig. 4, onde maiores quantidades de tensoativo são transferidas para a argila BG_3,16\%, pois a quantidade de tensoativo que não foi sorvida na argila BG_3,16\% e permaneceu na fase líquida foi de $4,648 \times 10^{3} \mathrm{mg} / \mathrm{L}$ (para o Teor 4); e, à medida que a concentração de argila foi aumentada, a quantidade de tensoativo que não foi sorvido $\left(\mathrm{C}_{\mathrm{e}}\right)$ também aumentou, chegando a $1,296 \times 10^{4} \mathrm{mg} / \mathrm{L}$ (Teor 4) para a argila BG_7,16\%, que como observado na Tabela III foi a que apresentou um menor valor de $\mathrm{K}_{\mathrm{d}}$ e um dos menores valores de $K_{f}$ ou seja, quanto maior a concentração de argila, menor é a transferência de tensoativo, pois aumentandose a concentração de argila o meio torna-se mais viscoso, dificultando o processo de difusão do tensoativo para os sítios de adsorção.

Por meio do gráfico da Fig. 5 fica evidenciado e confirmado que a argila que apresentou quantidade menor de tensoativo não sorvida foi a BG_3,16\%; desse fato infere-se que se menos tensoativo ficou na solução mais tensoativo foi sorvido, fato que pôde ser analisado pelo $\mathrm{K}_{\mathrm{d}}$, visto que esse é um coeficiente que traz informação sobre a distribuição do tensoativo da fase líquida para a argila (fase sólida). Vale ressaltar que o tipo de argila e o tipo de tensoativo são alguns dos fatores que influenciam o $\mathrm{K}_{\mathrm{d}}$, porém a argila que apresentou maior valor de $\mathrm{K}_{\mathrm{d}}$ foi a de concentração $3,16 \%$.

As isotermas de sorção da argila Brasgel com o tensoativo TA50 são apresentadas na Fig. 6. Analisando estas isotermas, observa-se que as argilas se ajustaram tanto ao modelo linear como de Freundlich. Com relação ao tensoativo sorvido na argila, observa-se um fato semelhante ao ocorrido quando se utilizou o tensoativo WB, que a quantidade de tensoativo sorvido cresce com o aumento da concentração inicial do mesmo, ou seja, quando a concentração de tensoativo adicionada inicialmente no processo de organofilização da argila é aumentada, a quantidade sorvida também aumenta para todas as concentrações de argilas. Vale destacar mais uma vez que o processo organofilização com TA 50 é diferente de quando se utiliza o WB; pelo fato do TA 50 ser um tensoativo não iônico, o mesmo é sorvido na argila por meio de adsorção. Os parâmetros desses modelos podem ser encontrados na Tabela IV.

Para a argila Brasgel que foi organofilizada com o TA 50 (Tabela IV), observa-se que todas as argilas foram ajustadas aos dois modelos, porém de acordo com o $\mathrm{R}^{2}$ as argilas BG_3,16\%,BG_4,16\%,BG_6,16\% e BG_7,16\% ajustaramse melhores ao modelo de Freundlich, enquanto a argila BG_5,16\% teve seus dados experimentais melhor ajustados ao modelo linear. $\mathrm{O}$ valor de $\mathrm{N}$ foi inferior a 1 , indicando que a sorção é desfavorável, porém vale salientar que $\mathrm{N}$ é um coeficiente do modelo de Freundlich e para argila BG_5,16\% os dados experimentais se ajustaram melhor ao modelo 
linear; porém, dentre os valores de $\mathrm{K}_{\mathrm{d}}$, a argila $\mathrm{BG}$ _5,16\% foi a que apresentou menor valor de $\mathrm{K}_{\mathrm{d}}$, consequentemente essa argila apresenta menor capacidade de sorção, quando comparada com as demais. Na Fig. 7, é possível observar de uma forma mais detalhada as informações obtidas pelo $\mathrm{K}_{\mathrm{d}}$ e $\mathrm{K}_{\mathrm{f}}$ Observa-se que a quantidade de tensoativo que não foi sorvida na argila foi um pouco irregular, ainda assim fica evidente que restou menos TA 50 na fase líquida quando foi utilizada a argila BG_3,16\%, ou seja, mais tensoativo ficou sorvido na argila, por isso o valor de $\mathrm{K}_{\mathrm{d}}$ foi maior para essa argila.

De maneira geral, ao aplicar os modelos com os parâmetros obtidos para cada ensaio, foi possível verificar e determinar qual dos modelos foi mais bem ajustado aos dados experimentais, onde de forma geral o modelo linear representou $70 \%$ das isotermas de sorção. Mas, vale salientar que tanto para a argila organofilizada com o WB quanto para a organofilizada com o TA 50, quando comparado os coeficientes de determinação do modelo linear e do Freundlich, a diferença entres eles é mínima. É importante ainda ressaltar que de acordo com os estudos mostrados a capacidade de sorção das argilas pode ser superior à capacidade de troca catiônica (CTC), fato este que depende de algumas propriedades características do tensoativo usado na síntese da argila organofílica. Vale ressaltar que quando se aumenta muito a concentração de tensoativo na organofilização formam-se camadas de tensoativo na interface da argila e este fato dificulta o processo de moagem.

\section{CONCLUSÕES}

Diante dos resultados que foram apresentados, pode-se inferir que: por meio da técnica de difração de raios $X$ foi possível caracterizar e confirmar a modificação química da argila bentonítica, visto que esta argila apresentou distância interplanar menor que as argilas organofilizadas; o método usado para verificar a quantidade de tensoativo sorvido na argila mostrou-se eficiente, pois foi possível aplicar os modelos linear e de Freundlich para obtenção das isotermas e dos respectivos parâmetros para cada ensaio, utilizando os dados fornecidos na análise termogravimétrica; ao analisar $\mathrm{K}_{\mathrm{d}}$, percebeu-se que é uma constante importante na quantificação da sorção de tensoativo, onde foi possível analisar em quais concentrações de argila e qual argila foi capaz de sorver mais os tensoativos estudados; através dos estudos sobre a sorção, foi possível inferir através do modelos de isotermas que o tensoativo foi sorvido na argila através do processo de adsorção em multicamadas e adsorção cooperativa. Por fim, podemos indicar que a concentração de argila de $3,16 \%$, tratada tanto com o tensoativo iônico WB quanto com o tensoativo não iônico TA 50, foi a que obteve a melhor capacidade de sorção.

\section{REFERÊNCIAS}

[1] L.B.Paiva, A.R. Morales, F.R.V. Díaz, Cerâmica 54 (2008) 213.
[2] P.S. Souza, Tecnologia das Argilas, $1^{\text {a }}$ Ed., USP/Edgard Blucher Ltda (1975).

[3] A. R.V. Silva, H.C. Ferreira, Rev. Eletr. Mater. Proc. 3.2 (2008) 26.

[4] J.J.B. Cardodo, "Estudo do inchamento de bentonitas sódicas e avaliação do desempenho de inibidores pela difração de raios X", Tese Dr., Universidade Federal do Rio de Janeiro, Brasil (2005).

[5] C.S. Ross, E.V. Shannon, J. Am. Ceram. Soc. 9 (1926) 77.

[6] D.C. Rodríguez-Sarmiento, J.A. Pinzón-Bello, Appl. Clay Sci. 18 (2001) 173.

[7] F.R. Valenzuela Diaz, "Preparação, a nível de laboratório, de algumas argilas esmectiticas organofilicas", Tese Dr., Universidade de S. Paulo, Brasil (1994).

[8] H.S. Ferreira; R.R. Menezes, A.B. Martins, H.C. Ferreira, Cerâmica 54 (2008) 77.

[9] H.S. Ferreira, L.F. Campos, R.R. Menezes, J.M. Cartaxo, L.N.L. Santana, G.A. Neves, H.C. Ferreira, Cerâmica 59 (2013) 277.

[10] I.A. Silva, F.K. A. Sousa, R.R. Menezes, G.A. Neves, L.N.L. Santana, H.C Ferreira, Appl. Clay Sci. 95 (2014) 371. [11] S.L.A. Dantas, K.A. Marques, H.S. Ferreira, Cerâmica 61 (2015) 199.

[12] C.W. Fetter, Contaminant Hidrogeology, $2^{\text {nd }}$ Ed., New Jersey, USA, Prentice Hall (1999).

[13] R.A. Freeze, J.A. Cherry, Groundwater, $1^{\text {th }}$ Ed., Prentice Hall (1979).

[14] M.B. Ferreira, "Estudo paramétrico do transporte e remediação de hpa em solo com o programa modflow", Diss. Mestrado, Universidade Federal do Rio de Janeiro, Brasil (2010).

[15] O. Zanella, "Sorção de Nitrato de Carvão Ativado Tratado com $\mathrm{CaCl}_{2}$ : Estudo de ciclos de sorção/ Regeneração", Diss. Mestrado, Universidade Federal do Rio Grande do Sul, Brasil (2012).

[16] A.T. Elbachá, "Estudo da Influência de Alguns Parâmetros no Transporte de Massa em Solos Argilosos", Diss. Mestrado, Pontifícia Universidade Católica do Rio de Janeiro, Brasil (1989).

[17] P.O.S. Costa, "Avaliação em laboratório, do transporte de contaminantes no solo do aterro sanitário de Sauípe/BA", Diss. Mestrado, Pontifícia Universidade Católica do Rio de Janeiro, Brasil (2002).

[18] T.J. Chen, P.S. Santos, H.C. Ferreira, A.R. Zandonadi, S.F. Calil, L.V. Campos, Cerâmica 20 (1974) 305.

[19] J.R.T. Fagundes, L.V. Zuquete, Rev. Bras. Geocienc. 39 (2009) 494.

[20] P.M. Naranjo, E.L. Sham, E.R. Castellón, R.M.T. Sánchez, E.M.F. Torres, Clay Miner. 61 (2013) 98.

[21] N.N. Varzacacou, "Interação de gasolina, benzeno, tolueno e xilenos com argilominerais esmectíticos da formação resende, bacia de São Paulo", Diss. Mestrado, Universidade de S. Paulo, Brasil (2009).

[22] B. Velde, Origin and Mineralogy of Clays, $1^{\text {st }}$ Ed., Springer (1995).

[23] S. Paria, C. Manohar, K.C. Khilar, Ind. Eng. Chem. 
Res. 44 (2005) 3091.

[24] C.G.F. T. Rossi, T.N. de C. Dantas, A.A.D. Neto, M.A.M. Maciel, Rev. Univ. Rural, Ser. Cienc. Exatas Terra 25 (2006) 73.
[25] M.H. Kalavathy, T. Karthikeyan, S. Rajgopal, L.R. Miranda, J. Colloid Interface Sci. 292 (2005) 354.

(Rec. 16/11/2015, Rev. 18/12/2015, 04/01/2016, 22/03/2016, Ac. 22/03/2016) 